\title{
INFINITESIMAL VARIATIONS OF INVARIANT SUBMANIFOLDS OF A SASAKIAN MANIFOLD
}

\author{
By Kentaro Yano, U-Hang Ki and Jin Suk PaK
}

\section{$\S 0$. Introduction.}

An infinitesimal variation of an invariant (complex) submanifold of a Kaehlerian manifold which carries it into an invariant submanifold is said to be complex. An infinitesimal variation is said to be holomorphic when it is complex and preserves the complex structure on the invariant submanifold. ([1], [4], [5]). Okumura and two of the present authors [5] proved that an infinitesimal complex conformal variation of a compact orientable submanifold of a Kaehlerian manifold is necessarily isometric and holomorphic and derived a necessary and sufficient condition for a complex variation to be volume-preserving and holomorphic by using an integral formula.

The main purpose of the present paper is to study infinitesimal variations of invariant submanifolds of a Sasakian manifold and to prove theorems analogous to those proved in [4] and [5].

In preliminary $\S 1$ we state some properties of invariant submanifolds of a Sasakian manifold.

In $\S 2$, we derive fundamental formulas in the theory of infinitesimal variations and study invariant variations, that is, infinitesimal variations which carry an invariant submanifold into an invariant submanifold. In $\$ 3$ we study $f$-preserving variations, that is, invariant variations which preserve the tensor field $f_{b}{ }^{a}$ of the Sasakian structure $\left(f_{b}{ }^{a}, g_{c b}, f_{b}\right)$ induced on an invariant submanifold.

In $\S 4$ we study invariant conformal variations and prove that an invariant conformal fiber-preserving variation of a compact orientable invariant submanifold of a Sasakian manifold is necessarily isometric and hence $f$-preserving. In the last $\$ 5$ we prove an integral formula concerning invariant variation and show some of its applications.

Received April 13, 1977. 


\section{$\S 1$. Invariant submanifolds of a Sasakian manifold.}

Let $M^{2 m+1}$ be a $(2 m+1)$-dimensional Sasakian manifold covered by a system of coordinate neighborhoods $\left\{U ; x^{h}\right\}$ and $\left(f_{\imath}{ }^{h}, g_{j i}, f_{\imath}\right)$ the set of structure tensors of $M^{2 m+1}$, where, here and in the sequel, the indices $h, i, j, \cdots$ run over the range $\left\{1^{\prime}, 2^{\prime}, \cdots,(2 m+1)^{\prime}\right\}$. Then we have

$$
f_{\imath}{ }^{t} f_{t}{ }^{h}=-\delta_{\imath}{ }^{h}+f_{\imath} f^{h}, \quad f_{t} f_{\imath}{ }^{t}=0, \quad f_{t}{ }^{h} f^{t}=0, \quad f_{t} f^{t}=1
$$

and

$$
f_{j}{ }^{t} f_{i}^{s} g_{t s}=g_{j i}-f_{j} f_{\imath},
$$

$f^{h}$ being the vector field associated with $f_{\imath}$, that is, $f^{h}=f_{\imath} g^{i n}, g^{i n}$ being contravariant metric tensor. We also have

$$
\nabla_{\imath} f^{h}=f_{\imath}{ }^{h}
$$

and

$$
\nabla_{j} f_{2}{ }^{h}=-g_{j i} f^{h}+\delta_{j}^{h} f_{\imath},
$$

where $\nabla_{\imath}$ denotes the operator of covariant differentiation with respect to $g_{j i}$.

Let $M^{2 n+1}(n<m)$ be a $(2 n+1)$-dimensional Riemannian manifold covered by a system of coordinate neighborhoods $\left\{V ; y^{a}\right\}$ and isometrically immersed in $M^{2 m+1}$ by the immersion $\imath ; M^{2 n+1} \rightarrow M^{2 m+1}$, where, here and in the sequel, the indices $a, b, c, \cdots$ run over the range $\{1,2, \cdots,(2 n+1)\}$. We identify $\imath\left(M^{2 n+1}\right)$ with $M^{2 n+1}$ and represent the immersion by $x^{h}=x^{h}\left(y^{a}\right)$. If we put $B_{b}^{h}=\partial_{b} x^{h}$ $\left(\partial_{b}=\partial / \partial y^{b}\right)$, then $B_{b}{ }^{h}$ are $2 n+1$ linearly independent vectors of $M^{2 m+1}$ tangent to $M^{2 n+1}$. Denoting by $g_{c b}$ the Riemannian metric tensor of $M^{2 n+1}$ we have $g_{c b}=$ $g_{j i} B_{c}{ }^{j} B_{c}{ }^{2}$ since the immersion is isometric. We denote by $C_{y}{ }^{n} 2(m-n)$ mutually orthogonal unit normals to $M^{2 n+1}$, then we have $g_{j i} B_{b}{ }^{j} C_{y}{ }^{2}=0$ and the metric tensor of the normal bundle of $M^{2 n+1}$ is given by $g_{z y}=g_{j i} C_{z}{ }^{j} C_{y}{ }^{2}=\delta_{z y}, \delta_{z y}$ being the Kronecker delta, where, here and in the sequel, the indices $u, v, x, y, z$ run over the range $\{(2 n+2), \cdots,(2 m+1)\}$.

We denote by $\Gamma_{j i}^{h}, \Gamma_{c b}^{a}$ and $\Gamma_{c y}^{x}$ the Christoffel symbols formed with $g_{j i}$, those formed with $g_{c b}$ and the components of the connection induced in the normal bundle of $M^{2 n+1}$, that is,

$$
\Gamma_{c y}^{x}=\left(\partial_{c} C_{y}{ }^{h}+\Gamma_{j i}^{h} B_{c}{ }^{j} C_{y}{ }^{i}\right) C^{x}{ }_{h}, \quad C^{x}{ }_{h}=C_{y}{ }^{2} g^{y x} g_{i h}
$$

respectively, $g^{y x}$ being the contravariant components of the metric tensor of the normal bundle. Then the van der Waerden-Bortolotti covariant derivatives of $B_{b}{ }^{h}$ and $C_{y}{ }^{h}$ are respectively given by

$$
\nabla_{c} B_{b}{ }^{h}=\partial_{c} B_{b}{ }^{h}+\Gamma_{j i}^{h} B_{c}{ }^{j} B_{b}{ }^{2}-\Gamma_{c b}^{a} B_{a}{ }^{h}
$$


and

$$
\nabla_{c} C_{y}{ }^{h}=\partial_{c} C_{y}{ }^{h}+\Gamma_{j i}^{h} B_{c}{ }^{j} C_{y}{ }^{2}-\Gamma_{c y}^{x} C_{x}{ }^{h},
$$

and the equations of Gauss and Weingarten are respectively

$$
\nabla_{c} B_{b}^{h}=h_{c b}{ }^{x} C_{x}^{h} \text { and } \nabla_{c} C_{y}^{h}=-h_{c}^{a} B_{a}^{h},
$$

where $h_{c b}{ }^{x}$ are components of the second fundamental tensors of $M^{2 n+1}$ and $h_{c}^{a}{ }_{y}=h_{c b}{ }^{z} g^{b a} g_{z y}, g^{b a}$ being contravariant components of the metric tensor of $M^{2 n+1}$.

Denoting by $K_{k j i}{ }^{h}, K_{d c b}{ }^{a}$ and $K_{d c y}{ }^{x}$ the curvature tensors of $M^{2 m+1}, M^{2 n+1}$ and the normal bundle of $M^{2 n+1}$, we have the following equations of Gauss, Codazzi and Ricci respectively:

$$
\begin{gathered}
K_{d c b}{ }^{a}=K_{k j i}{ }^{h} B_{d c b h}^{k j i a}+h_{d}{ }^{a}{ }_{x} h_{c b}{ }^{x}-h_{c}{ }^{a}{ }_{x} h_{d b}{ }^{x}, \\
0=K_{k j l}{ }^{h} B_{a c b}^{k j i} C^{x}{ }_{h}-\left(\nabla_{d} h_{c b}{ }^{x}-\nabla_{c} h_{d b}{ }^{x}\right)
\end{gathered}
$$

and

$$
K_{d c y}{ }^{x}=B_{d c}^{k j} C_{y}{ }^{2} C^{x}{ }_{h}+\left(h_{d e}{ }^{x} h_{c}{ }^{e} y-h_{c e}{ }^{x} h_{d y}{ }^{e} y\right),
$$

where $B_{d c b h}^{k j i a}=B_{d}{ }^{k} B_{c}{ }^{j} B_{b}{ }^{\imath} B^{a}{ }_{h}, B_{d c b}^{k j j}=B_{d}{ }^{k} B_{c}{ }^{\jmath} B_{b}{ }^{\imath}$ and $B_{d c}^{k j}=B_{d}{ }^{k} B_{c}{ }^{\jmath}$.

A $(2 n+1)$-dimensional submanifold $M^{2 n+1}$ is called an invariant submanifold of the Sasakian manifold $M^{2 m+1}$ if the tangent space at each point of $M^{2 n+1}$ is invariant under the action of $f_{\imath}{ }^{h}$. Thus for an invariant submanifold $M^{2 n+1}$, we have

$$
f_{\imath}{ }^{h} B_{b}{ }^{\imath}=f_{b}^{a} B_{a}^{h}, \quad f_{\imath}{ }^{h} C_{y}{ }^{\imath}=f_{y}{ }^{x} C_{x}{ }^{h},
$$

$f_{b}{ }^{a}$ and $f_{y}{ }^{x}$ being tensor fields of type (1.1) of $M^{2 n+1}$ and the normal bundle of $M^{2 n+1}$ respectively. Putting $f_{b a}=f_{b}{ }^{e} g_{e a}$ and $f_{y x}=f_{y}{ }^{z} g_{z x}$, we have $f_{b a}=f_{j i} B_{b a}^{i i}$ and $f_{y x}=f_{j i} C_{y}{ }^{3} C_{x}{ }^{2}$ and consequently

$$
f_{b a}=-f_{a b}, \quad f_{y x}=-f_{x y} .
$$

On the other hand, we put

$$
f^{h}=f^{a} B_{a}{ }^{h}+f^{x} C_{x}{ }^{h} .
$$

Now applying the operator $f_{h}{ }^{k}$ to the first equation of (1.10) and using (1.1) and (1.11), we find

$$
-B_{b}{ }^{k}+f_{b}\left(f^{a} B_{a}{ }^{k}+f^{x} C_{x}{ }^{k}\right)=f_{b}{ }^{e} f_{e}^{a} B_{a}{ }^{k},
$$

where $f_{b}=f^{c} g_{c b}$, from which

$$
f_{b}{ }^{e} f_{e}^{a}=-\delta_{b}^{a}+f_{b} f^{a}, \quad f_{b} f^{x}=0 .
$$


Since $M^{2 n+1}$ is odd dimensional, we see from the first equation of (1.12) that $f_{b}$ never vanishes and consequently from the second equation of (1.12) we see that $f^{x}=0$ and consequently (1.11) becomes

$$
f^{h}=f^{a} B_{a}^{h},
$$

which shows that $f^{h}$ is tangent to the invariant submanifold. From (1.1), (1.10) and (1.13) we find

$$
f_{b}^{a} f^{b}=0, \quad f_{a} f^{a}=1 .
$$

Also transvecting (1.2) with $B_{c}{ }^{\jmath} B_{b}{ }^{2}$, we find

$$
f_{c}^{e} f_{b}{ }^{d} g_{e d}=g_{c b}-f_{c} f_{b} .
$$

Equations (1.12), (1.14) and (1.15) show that the invariant submanifold $M^{2 n+1}$ admits an almost contact metric structure.

Applying the operator $f_{h}{ }^{k}$ to the second equation of (1.10) and using (1.1) and (1.13), we find

$$
-C_{y}{ }^{k}=f_{y}{ }^{z} f_{z}^{x} C_{x}{ }^{k},
$$

from which

$$
f_{y}{ }^{z} f_{z}^{x}=-\delta_{y}^{x}
$$

From (1.2), we have

$$
f_{z}^{v} f_{y}^{u} g_{v u}=g_{z y}
$$

and consequently $f_{y}{ }^{x}$ defines an almost Hermitian structure in the normal bundle.

Now differentiating (1.13) covariantly along $M^{2 n+1}$ and using (1.3) and (1.6), we find

$$
f_{\imath}{ }^{n} B_{b}{ }^{2}=\left(\nabla_{b} f^{a}\right) B_{a}{ }^{h}+f^{a} h_{b a}{ }^{x} C_{x}{ }^{n},
$$

from which

$$
\nabla_{b} f^{a}=f_{b}{ }^{a}
$$

and

$$
h_{c b}^{x} f^{b}=0 \text {. }
$$

Also differentiating the first equation of (1.10) covariantly and substituting (1.4) and (1.6), we find

$$
\left(-g_{j i} f^{h}+\delta_{j}^{h} f_{\imath}\right) B_{c b}^{j i}+f_{\imath}{ }^{h} h_{c b}{ }^{x} C_{x}{ }^{2}=\left(\nabla_{c} f_{b}{ }^{a}\right) B_{a}{ }^{h}+f_{b}{ }^{e} h_{c e}{ }^{x} C_{x}{ }^{h},
$$

from which, taking account of (1.10) and (1.13) 


$$
\begin{gathered}
\nabla_{c} f_{b}^{a}=-g_{c b} f^{a}+\delta_{c}^{a} f_{b}, \\
h_{c b}{ }^{y} f_{y}{ }^{x}=h_{c e}{ }^{x} f_{b}{ }^{e} .
\end{gathered}
$$

From (1.21) we find $g^{c b} h_{c b}^{y} f_{y}^{x}=0$, from which, $f_{y}^{x}$ defining an almost complex structure,

$$
g^{c b} h_{c b}^{y}=0,
$$

which shows that an invariant submanifold $M^{2 n+1}$ is minimal.

Equations (1.18) and (1.20) show that the almost contact metric structure induced on the invariant submanifold is Sasakian.

Differentiating the second equation of (1.10) covariantly, we find

$$
\left(-g_{j i} f^{h}+\delta_{j}^{h} f_{\imath}\right) B_{c}{ }^{j} C_{y}{ }^{2}-f_{\imath}{ }^{h} h_{c}{ }^{a}{ }_{y} B_{a}{ }^{\imath}=\left(\nabla_{c} f_{y}{ }^{x}\right) C_{x}{ }^{h}-f_{y}{ }^{x} h_{c}{ }^{a}{ }_{x} B_{a}{ }^{h},
$$

from which, using (1.10) and (1.21),

$$
\nabla_{c} f_{y}^{x}=0,
$$

which shows that the almost Hermitian structure in the normal bundle is covariantly constant.

We close this section by preparing some formulas for later use. It is known that on a Sasakian manifold the following identities are valid:

$$
\frac{1}{2} K_{d c b a} f^{d c}=K_{b c} f_{a}^{c}+(2 n-1) f_{b a},
$$

$$
K_{b e} f_{a}^{e}+K_{a e} f_{b}^{e}=0
$$

$$
K_{b e} f^{e}=2 n f_{b},
$$

where $K_{d c b a}=K_{d c b}{ }^{e} g_{e a}, K_{c b}=K_{e c b}{ }^{e}$ and $f^{d c}=g^{d e} f_{e}^{c}$. Transvecting (1.24) with $f_{e}^{b}$ and using (1.1), we find

$$
\frac{1}{2} K_{d c b a} f^{d c} f_{e}^{b}=K_{b c} f_{e}^{b} f_{a}^{c}+(2 n-1)\left(-g_{e a}+f_{e} f_{a}\right),
$$

from which, using (1.25),

$$
\frac{1}{2} K_{d c b a} f^{d c} f_{e}^{b}=K_{e a}-\left(K_{e d} f^{d}\right) f_{a}+(2 n-1)\left(-g_{e a}+f_{e} f_{a}\right)
$$

or

$$
\frac{1}{2} K_{d c b e} f^{d c} f_{a}^{e}=K_{b a}-(2 n-1) g_{b a}-f_{b} f_{a}
$$




\section{$\S 2$. Infinitesimal variations of invariant submanifolds.}

Let $M^{2 n+1}$ be a $(2 n+1)$-dimensional invariant submanifold of a $(2 m+1)$ dimensional Sasakian manifold $M^{2 m+1}$. We consider an infinitesimal variation of $M^{2 n+1}$ of $M^{2 m+1}$ given by

$$
\bar{x}^{h}=x^{h}+v^{h}(y) \varepsilon,
$$

where $\varepsilon$ is an infinitesimal. Putting $\bar{B}_{b}{ }^{h}=\partial_{b} \bar{x}^{h}$, we have

$$
\bar{B}_{b}{ }^{h}=B_{b}{ }^{h}+\left(\partial_{b} v^{h}\right) \varepsilon,
$$

which are $2 n+1$ linearly independent vectors tangent to the varied submanifold at $\left(\bar{x}^{h}\right)$. We displace $\bar{B}_{b}{ }^{h}$ back parallelly from $\left(\bar{x}^{h}\right)$ to $\left(x^{h}\right)$, then we have

$$
\tilde{B}_{b}{ }^{h}=\bar{B}_{b}{ }^{h}+\Gamma_{j i}^{h}(x+v \varepsilon) v^{\jmath} \bar{B}_{b}{ }^{2} \varepsilon .
$$

Thus putting $\delta B_{b}{ }^{h}=\tilde{B}_{b}{ }^{h}-B_{b}{ }^{h}$, we obtain

$$
\delta B_{b}{ }^{h}=\left(\nabla_{b} v^{h}\right) \varepsilon,
$$

neglecting terms of order higher than one with respect to $\varepsilon$, where

$$
\nabla_{b} v^{h}=\partial_{b} v^{h}+\Gamma_{j i}^{h} B_{b} v^{2} .
$$

In the sequel we always neglect terms of order higher than one with respect to $\varepsilon$.

On the other hand, putting

$$
v^{h}=v^{a} B_{a}^{h}+v^{x} C_{x}^{h},
$$

we have

$$
\nabla_{b} v^{h}=\left(\nabla_{b} v^{a}-h_{b}{ }^{a}{ }_{x} v^{x}\right) B_{a}{ }^{h}+\left(\nabla_{b} v^{x}+h_{b a}{ }^{x} v^{a}\right) C_{x}{ }^{h} .
$$

Thus (2.3) can be written as

$$
\delta B_{b}{ }^{h}=\left[\left(\nabla_{b} v^{a}-h_{b}{ }^{a}{ }_{x} v^{x}\right) B_{a}{ }^{h}+\left(\nabla_{b} v^{x}+h_{b a}{ }^{x} v^{a}\right) C_{x}{ }^{h}\right] \varepsilon .
$$

When the tangent space at a point $\left(x^{h}\right)$ of the submanifold and that at the corresponding point $\left(\bar{x}^{h}\right)$ of the varied submanifold are always parallel, the variation is said to be parallel [3]. Hence (2.7) implies

LEMMA 2.1. ([3]) In order for an infinttesimal variation of a submanifold to be parallel, it is necessary and sufficient that

$$
\nabla_{b} v^{x}+h_{b a} v^{a}=0
$$


We now assume that the infinitesimal variation (2.1) carries an invariant submanifold into an invariant submanifold and call such a variation an invariant variation. For an invariant variation, $f_{\imath}{ }^{h}(x+v \varepsilon) \bar{B}_{b}{ }^{2}$ are linear combinations of $\bar{B}_{b}{ }^{h}$ and vice versa.

Now we have

$$
\begin{aligned}
f_{\imath}{ }^{h}(x & +v \varepsilon) \bar{B}_{b}{ }^{\imath} \\
= & {\left[f_{\imath}{ }^{h}+v^{\jmath} \partial_{\jmath} f_{\imath}{ }^{h} \varepsilon\right]\left[B_{b}{ }^{2}+\partial_{b} v^{\imath} \varepsilon\right] } \\
= & f_{\imath}{ }^{h} B_{b}{ }^{2}+v^{j}\left[-\Gamma_{j t}^{h} f_{\imath}{ }^{t}+\Gamma_{j i}^{t} f_{t}{ }^{h}-g_{j i} f^{h}+\delta_{\jmath}^{h} f_{\imath}\right] B_{b}{ }^{i} \varepsilon+f_{\imath}{ }^{h} \partial_{b} v^{\imath} \varepsilon \\
= & f_{b}{ }^{a} \bar{B}_{a}{ }^{h}-f_{b}{ }^{a}\left(\partial_{a} v^{h}\right) \varepsilon+f_{\imath}{ }^{h}\left(\partial_{b} v^{\imath}+\Gamma_{j t}^{i} B_{b}{ }^{\jmath} v^{t}\right) \varepsilon-f_{b}{ }^{a} \Gamma_{j i}^{h} v^{\jmath} B_{a}{ }^{\imath} \varepsilon \\
& +f_{b}\left(v^{a} B_{a}{ }^{h}+v^{x} C_{x}{ }^{h}\right) \varepsilon-v_{b} f^{a} B_{a}{ }^{h} \varepsilon \\
= & f_{b}{ }^{a} \bar{B}_{a}{ }^{h}+f_{\imath}{ }^{h} \nabla_{b} v^{\imath} \varepsilon-f_{b}{ }^{a} \nabla_{a} v^{h} \varepsilon+\left(f_{b} v^{a}-v_{b} f^{a}\right) B_{a}{ }^{h} \varepsilon+f_{b} v^{x} C_{x}{ }^{h} \varepsilon,
\end{aligned}
$$

where we have used (1.4), (1.13), (2.2), (2.4) and (2.5), and consequently from (2.6)

$$
\begin{aligned}
f_{\imath}{ }^{h}(x & +v \varepsilon) \vec{B}_{b}{ }^{2} \\
= & {\left[f_{b}{ }^{a}+\left\{f_{e}{ }^{a}\left(\nabla_{b} v^{e}-h_{b}{ }^{e} x^{x}\right)-f_{b}{ }^{e}\left(\nabla_{e} v^{a}-h_{e}{ }^{a}{ }_{x} v^{x}\right)\right.\right.} \\
& \left.\left.+f_{b} v^{a}-v_{b} f^{a}\right\} \varepsilon\right] \bar{B}_{a}{ }^{h} \\
& +\left[\left(\nabla_{b} v^{y}+h_{b a}{ }^{y} v^{a}\right) f_{y}{ }^{x}-f_{b}{ }^{e}\left(\nabla_{e} v^{x}+h_{e a}{ }^{x} v^{a}\right)+f_{b} v^{x}\right] \bar{C}_{x}{ }^{h} \varepsilon,
\end{aligned}
$$

where $\bar{C}_{x}{ }^{h}$ denote $2(m-n)$ mutually orthogonal unit normals to the varied submanifold and $v_{b}=g_{b a} v^{a}$. Hence we have, using (1.21),

PROPOSITION 2.2. In order for an infinitesimal variation of an invariant submanifold to be invariant it is necessary and sufficient that

$$
\left(\nabla_{b} v^{y}+h_{b a}^{y} v^{a}\right) f_{y}^{x}-f_{b}^{e}\left(\nabla_{e} v^{x}+h_{e a}^{x} v^{a}\right)+f_{b} v^{x}=0
$$

or

$$
\left(\nabla_{b} v^{y}\right) f_{y}^{x}-f_{b}^{e} \nabla_{e} v^{x}+f_{b} v^{x}=0 .
$$

When $v^{x}=0$, that is, when the variation vector $v^{h}$ is tangent to the submanifold, the variation is said to be tangential and when $v^{a}=0$, that is, when the variation vector $v^{h}$ is normal to the submanifold, the variation is said to be normal [3]. From Proposition 2.2, we have

Corollary 2.3. A tangential variation of an invariant submanifold is invariant. 
Combining Proposition 2.2 and Lemma 2.1, we also have

COROLlary 2.4. In order for a parallel variation of an invariant submanifold to be invariant, it is necessary and sufficient that the variation is tangentıal.

Now applying the operator $\delta$ to $g_{c b}=g_{j i} B_{c}{ }^{\jmath} B_{b}{ }^{2}$ and using $\delta g_{j i}=0$ and (2.7), we find

$$
\delta g_{c b}=\left[\nabla_{c} v_{b}+\nabla_{b} v_{c}-2 h_{c b x} v^{x}\right] \varepsilon,
$$

from which

$$
\delta g^{b a}=-\left[\nabla^{b} v^{a}+\nabla^{a} v^{b}-2 h^{b a}{ }_{x} v^{x}\right] \varepsilon,
$$

where $\nabla^{b}=g^{b a} \nabla_{a}$ and $h^{b a}{ }_{x}=g^{b e} g^{a d} h_{e d x}$.

An infinitesimal variation for which $\delta g_{c b}=0$ is said to be isometruc [1], [3].

We now put

$$
\bar{\Gamma}_{c b}^{a}=\left(\partial_{c} \bar{B}_{b}{ }^{h}+\Gamma_{j i}^{h}(\bar{x}) \bar{B}_{c}{ }^{j} \bar{B}_{b}{ }^{i}\right) \bar{B}^{a}{ }_{h}
$$

and

$$
\delta \Gamma_{c b}^{a}=\bar{\Gamma}_{c b}^{a}-\Gamma_{c b}^{a},
$$

where $\bar{\Gamma}_{c b}^{a}$ are Christoffel symbols of the varied submanifold.

Substituting (2.2) into (2.14), we find by a straightforward computation

$$
\delta \Gamma_{c b}^{a}=\left[\left(\nabla_{c} \nabla_{b} v^{a}+K_{k j i}{ }^{h} v^{k} B_{c b}^{j i}\right) B^{a}{ }_{h}+h_{c b}{ }^{x}\left(\nabla^{a} v_{x}+h_{d}{ }^{a}{ }_{x} v^{d}\right)\right] \varepsilon,
$$

from which, using equations of Gauss and Codazzi [3],

$$
\begin{aligned}
\delta \Gamma_{c b}^{a}= & \left(\nabla_{c} \nabla_{b} v^{a}+K_{d c b}{ }^{a} v^{d}\right) \varepsilon-\left[\nabla_{c}\left(h_{b e x} v^{x}\right)+\nabla_{b}\left(h_{c e x} v^{x}\right)\right. \\
& \left.-\nabla_{e}\left(h_{c b x} v^{x}\right)\right] g^{e a} \varepsilon .
\end{aligned}
$$

An infinitesimal variation of a submanifold for which $\delta \Gamma_{c b}^{a}=0$ is said to be affine.

We now prove

LEMMA 2.5. If an infinitesimal variation of an invariant submanifold is isometruc, then we have

$$
\begin{gathered}
g^{c b} \nabla_{c} \nabla_{b} v_{a}+\frac{1}{2} K_{d c e b} f_{a}^{e} f^{d c} v^{b}-2 \nabla^{e}\left(h_{e a}{ }^{x} v_{x}\right) \\
=-(2 n-1) v_{a}-\left(f_{b} v^{b}\right) f_{a} .
\end{gathered}
$$

Proof. Since an isometric variation is affine, we have from (2.17)

$$
\nabla_{c} \nabla_{b} v_{a}+K_{d c b a} v^{d}-\nabla_{c}\left(h_{b a x} v^{x}\right)-\nabla_{b}\left(h_{c a x} v^{x}\right)+\nabla_{a}\left(h_{c b x} v^{x}\right)=0
$$


from which

$$
g^{c b} \nabla_{c} \nabla_{b} v_{a}+K_{d a} v^{d}-2 \nabla^{e}\left(h_{e a x} v^{x}\right)=0,
$$

which, together with (1.27), proves the lemma.

\section{§ 3. Infinitesimal $\boldsymbol{f}$-preserving variations.}

Suppose that an infinitesimal variation of an invariant submanifold is invariant. Then putting

$$
f_{\imath}^{h}(x+v \varepsilon) \bar{B}_{b}{ }^{2}=\left(f_{b}{ }^{a}+\delta f_{b}{ }^{a}\right) \bar{B}_{a}{ }^{h},
$$

we have, from (2.9),

$$
\delta f_{b}{ }^{a}=\left[f_{e}{ }^{a}\left(\nabla_{b} v^{e}-h_{b}{ }^{e} v^{x}\right)-f_{b}{ }^{e}\left(\nabla_{e} v^{a}-h_{e}{ }^{a}{ }_{x} v^{x}\right)+\left(f_{b} v^{a}-v_{b} f^{a}\right)\right] \varepsilon .
$$

When an infinitesimal invariant variation satisfies $\delta f_{b}^{a}=0$, we say that the invariant variation is f-preserving. From (1.20), (1.21) and (3.2) we have

LEMMA 3.1. In order for an infinitesimal variation to be f-preserving, it is necessary and sufficient that the variation satisfies (2.10) or (2.11) and

$$
\left(\nabla_{b} v^{e}\right) f_{e}^{a}-f_{b}^{e}\left(\nabla_{e} v^{a}\right)-2 h_{b}{ }^{e} v^{x} f_{e}^{a}+\left(f_{b} v^{a}-v_{b} f^{a}\right)=0
$$

or equivalently

$$
\mathcal{L} f_{b}{ }^{a}-2 h_{b}{ }^{e}{ }_{x} v^{x} f_{e}{ }^{a}=0,
$$

$\mathcal{L}$ denoting the Lie dervation with respect to $v^{a}$.

Now applying the operator $\delta$ to $f_{b}{ }^{a} f^{b}=0$, we find

$$
\left(\delta f_{b}{ }^{a}\right) f^{b}+f_{b}{ }^{a}\left(\delta f^{b}\right)=0,
$$

from which, substituting (3.2) and taking account of (1.19),

$$
\left[f_{e}^{a}\left(f^{b} \nabla_{b} v^{e}\right)+v^{a}-\left(v_{b} f^{b}\right) f^{a}\right] \varepsilon+f_{b}{ }^{a}\left(\delta f^{b}\right)=0
$$

or, using (1.12) and (1.18)

$$
\delta f^{a}=\left[\mathcal{L} f^{a}+\alpha f^{a}\right] \varepsilon
$$

for a certain function $\alpha$. On the other hand, applying the operator $\delta$ to $g_{c b} f^{c} f^{b}$ $=1$ and using (2.12)

$$
\left[\mathcal{L} g_{c b}-2 h_{c b x} v^{x}\right] f^{c} f^{b} \varepsilon+2 g_{c b}\left(\delta f^{c}\right) f^{b}=0,
$$

from which, using (1.19) and the above equation, $\alpha=0$ and consequently we have 


$$
\delta f^{a}=\left(\mathcal{L} f^{a}\right) \varepsilon .
$$

We now define a tensor field $T_{c b}$ by

$$
\begin{gathered}
T_{c b}=\nabla_{c} v_{b}-\left(\nabla_{e} v_{d}\right) f_{c}{ }^{e} f_{b}{ }^{d}-2 h_{c b}{ }^{x} v_{x}+f_{c} f_{b}\left(\nabla_{e} v_{d}\right) f^{e} f^{d} \\
+f_{c}\left(f_{b}{ }^{e} v_{e}\right)-f_{b}\left(f_{c}{ }^{e} v_{e}\right)
\end{gathered}
$$

and prove

LEMMA 3.2. In order for an infinitesimal isometric invariant variation of an invariant submanifold to be f-preserving, it is necessary and sufficient that $T_{c b}=0$.

Proof. Suppose that an infinitesimal invariant variation of an invariant submanifold is $f$-preserving. Then by Lemma 3.1 , we have

$$
\left(\nabla_{b} v^{e}\right) f_{e}{ }^{a}-f_{b}{ }^{e}\left(\nabla_{e} v^{a}\right)-2 h_{b}{ }^{e} v^{x} f_{e}{ }^{a}+\left(f_{b} v^{a}-v_{b} f^{a}\right)=0 .
$$

Transvecting this with $f_{a}^{c}$ and taking account of (1.12) and (1.19), we find

$$
\nabla_{b} v^{c}-\left(\nabla_{e} v_{d}\right) f_{b}^{e} f^{c d}-2 h_{b}{ }^{c} v^{x}-f_{e}\left(\nabla_{b} v^{e}\right) f^{c}-f_{b}\left(f_{e}^{c} v^{e}\right)=0,
$$

from which, transvecting with $f^{b}$,

$$
f^{e} \nabla_{e} v^{c}=f^{c}\left(\nabla_{e} v_{d}\right) f^{e} f^{d}+f_{e}^{c} v^{e},
$$

and consequently, using $\nabla_{c} v_{b}+\nabla_{b} v_{c}-2 h_{c b x} v^{x}=0$ which means that the variation is isometric,

$$
f_{e} \nabla_{c} v^{e}=-f_{c}\left(\nabla_{e} v_{d}\right) f^{e} f^{a}+f_{c}^{e} v_{e}
$$

Thus (3.7) becomes

$$
\begin{gathered}
\nabla_{b} v_{c}-\left(\nabla_{e} v_{d}\right) f_{b}^{e} f_{c}^{d}-2 h_{b c}{ }^{x} v_{x}+f_{b} f_{c}\left(\nabla_{e} v_{d}\right) f^{e} f^{d} \\
-\left(f_{b}^{e} v_{e}\right) f_{c}+\left(f_{c}^{e} v_{e}\right) f_{b}=0,
\end{gathered}
$$

which means $T_{c b}=0$.

Conversely we suppose that $T_{c b}=0$. Then we have

$$
\begin{gathered}
\nabla_{c} v^{b}+\left(\nabla_{e} v^{d}\right) f_{c}{ }^{e} f_{d}{ }^{b}-2 h_{c}{ }^{b}{ }_{x} v^{x}+f_{c} f^{b}\left(\nabla_{e} v_{d}\right) f^{e} f^{d} \\
-\left(f_{c}{ }^{e} v_{e}\right) f^{b}-\left(f_{e}{ }^{b} v^{e}\right) f_{c}=0 .
\end{gathered}
$$

Transvecting this with $f_{b}^{a}$ and using (1.12), we find

$$
\begin{gathered}
\left(\nabla_{c} v^{e}\right) f_{e}{ }^{a}-\left(\nabla_{e} v^{a}\right) f_{c}{ }^{e}-2 h_{c}{ }^{e}{ }_{x} v^{x} f_{e}{ }^{a}+v^{a} f_{c}+f_{d}\left(\nabla_{e} v^{d}\right) f_{c}{ }^{e} f^{a} \\
-f_{c} f^{a}\left(f_{e} v^{e}\right)=0 .
\end{gathered}
$$


Transvecting the same equation with $f_{b}$, we have

$$
\left(\nabla_{c} v^{b}\right) f_{b}+f_{c}\left(\nabla_{e} v_{d}\right) f^{e} f^{d}-f_{c}{ }^{e} v_{e}=0
$$

Therefore substituting (3.9) into (3.8), we obtain

$$
\left(\nabla_{c} v^{e}\right) f_{e}^{a}-\left(\nabla_{e} v^{a}\right) f_{c}^{e}-2 h_{c}{ }^{e} x v^{x} f_{e}{ }^{a}+f_{c} v^{a}-v_{c} f^{a}=0 .
$$

Thus Lemma 3.1 and this equation prove the lemma.

We next prove

LEMMA 3.3. For an infinitesimal isometric invariant variation of an invariant submanifold, we have

$$
\begin{gathered}
T_{c b}+T_{b c}=0, \\
T_{c b}+T_{e d} f_{c}{ }^{e} f_{b}{ }^{d}=\left(\mathcal{L} f_{c}\right) f_{b}-\left(\mathcal{L} f_{b}\right) f_{c}
\end{gathered}
$$

and

$$
T^{c b} T_{c b}=2 T^{c b} \nabla_{c} v_{b}-2\left(\mathcal{L} f^{e}\right)\left(\mathcal{L} f_{e}\right)-4\left(\mathcal{L} f^{e}\right)\left(f_{e}{ }^{d} v_{d}\right),
$$

where $T^{c b}=T_{e d} g^{e c} g^{d b}$.

Proof. For an isometric variation, we have from (2.12),

$$
\nabla_{b} v_{a}+\nabla_{a} v_{b}=2 h_{b a}{ }^{x} v_{x}
$$

from which, taking account of (1.19),

$$
\left(\nabla_{b} v_{a}\right) f^{b} f^{a}=0
$$

On the other hand, from (1.21), we have

$$
h_{c b}{ }^{x}=-h_{e d}^{x} f_{c}^{e} f_{b}^{d} .
$$

Thus, from the definition (3.6) of $T_{c b}$, we see that $T_{c b}+T_{b c}=0$.

On the other hand, using (3.14) and (3.15), we have, by a straightforward computation,

$$
T_{e d} f_{c}{ }^{e} f_{b}{ }^{d}=\left(\nabla_{e} v_{d}\right) f_{c}{ }^{e} f_{b}{ }^{d}-\nabla_{c} v_{b}+\left(\nabla_{c} v_{e}\right) f^{e} f_{b}+\left(\nabla_{e} v_{b}\right) f^{e} f_{c}+2 h_{c b}{ }^{x} v_{x}
$$

But

$$
\mathcal{L} f_{b}=v^{e} f_{e b}+f_{e} \nabla_{b} v^{e}
$$

and consequently (3.16) can be written as

$$
\begin{aligned}
T_{e d} f_{c}{ }^{e} f_{b}{ }^{d}= & -\left\{\nabla_{c} v_{b}-\left(\nabla_{e} v_{d}\right) f_{c}{ }^{e} f_{b}{ }^{d}-2 h_{c b}{ }^{x} v_{x}+f_{c}\left(f_{b}{ }^{e} v_{e}\right)-f_{b}\left(f_{c}{ }^{e} v_{e}\right)\right\} \\
& -f_{c} \mathcal{L} f_{b}+f_{b} \mathcal{L} f_{c} .
\end{aligned}
$$


Thus, taking account of (3.6) and (3.14), we find (3.11). Finally from the definition (3.6) of $T_{c b}$ and (3.10) we have

$$
T^{c b} T_{c b}=T^{c b}\left\{\nabla_{c} v_{b}-\left(\nabla_{e} v_{d}\right) f_{c}^{e} f_{b}{ }^{d}+f_{c}\left(f_{b}^{e} v_{e}\right)-f_{b}\left(f_{c}^{e} v_{e}\right)\right\} .
$$

Thus taking account of (3.11) and of

$$
T_{e b} f^{e}=-\mathcal{L} f_{b}=-f^{e} T_{b e},
$$

we have

$$
T^{c b} T_{c b}=2 T^{c b} \nabla_{c} v_{b}-2\left(\mathcal{L} f^{e}\right)\left(\mathcal{L} f_{e}\right)-4\left(\mathcal{L} f^{e}\right)\left(f_{e}^{d} v_{d}\right)
$$

and consequently the lemma is proved.

Now applying the operator $\nabla^{c}$ to (3.6) with (3.14) and using (1.18), (1.20) and (3.13), we see that

$$
\begin{aligned}
\nabla^{c} T_{c b}= & \nabla^{c} \nabla_{c} v_{b}-\frac{1}{2}\left(\nabla_{c} \nabla_{e} v_{d}-\nabla_{e} \nabla_{c} v_{d}\right) f^{c e} f_{b}{ }^{d}-2 \nabla^{c}\left(h_{c b}{ }^{x} v_{x}\right) \\
& -v_{b}+(2 n+1)\left(f^{e} v_{e}\right) f_{b}+2 n\left(\nabla_{e} v_{d}\right) f^{e} f_{b}{ }^{d},
\end{aligned}
$$

from which, using the Ricci identity and Lemma 2.5,

$$
\nabla^{c} T_{c b}=2 n\left\{\left(f^{c} v_{c}\right) f_{b}-v_{b}+\left(\nabla_{e} v_{d}\right) f^{e} f_{b}{ }^{d}\right\} .
$$

Hence taking account of

$$
f_{c} \mathcal{L} f_{b}^{c}=\left(f^{c} v_{c}\right) f_{b}-v_{b}+\left(\nabla_{e} v_{c}\right) f^{e} f_{b}^{c},
$$

we obtain

$$
\nabla^{c} T_{c b}=2 n\left(f_{c} \mathcal{L} f_{b}^{c}\right)=-2 n\left(f_{b}^{c} \mathcal{L} f_{c}\right) .
$$

Thus, substituting (3.12) and (3.17) into the identity:

$$
\nabla^{c}\left(T_{c b} v^{b}\right)=\left(\nabla^{c} T_{c b}\right) v^{b}+T_{c b} \nabla^{c} v^{b},
$$

we have

$$
\nabla^{c}\left(T_{c b} v^{b}\right)=\frac{1}{2} T^{c b} T_{c b}+\left(\mathcal{L} f^{e}\right)\left(\mathcal{L} f_{e}\right)+2(n+1)\left(\mathcal{L} f^{e}\right)\left(f_{e}^{d} v_{d}\right)
$$

Now an infinitesimal variation which satisfies $\mathcal{L} f^{b}=\alpha f^{b}, \alpha$ being a certain function, is said to be fiber-preserving. Thus for a fiber-preserving variation, we have

$$
\nabla^{c}\left(T_{c b} v^{b}\right)=\frac{1}{2} T^{c b} T_{c b}+\alpha^{2},
$$

from which, if the submanifold is compact orientable we have 


$$
\int\left[\frac{1}{2} T^{c b} T_{c b}+\alpha^{2}\right] d V=0
$$

$d V$ being the volume element of $M^{2 n+1}$. Thus we have

Proposition 3.4. If an infinitesimal isometric invariant variation of a compact orientable invariant submanfold of a Sasakian manifold is fiber-preserving, then it is f-preserving.

\section{$\S 4$. Infinitesimal conformal variations.}

An infinitesimal variation of a submanifold for which $\delta g_{c b}$ is proportional to $g_{c b}$ is said to be conformal. A necessary and sufficient condition for an infinitesimal variation (2.1) of a submanifold to be conformal is

$$
\nabla_{c} v_{b}+\nabla_{b} v_{c}-2 h_{c b}^{x} v_{x}=2 \lambda g_{c b},
$$

where

$$
\lambda=(1 /(2 n+1))\left(\nabla_{e} v^{e}-h_{e}^{e} x v^{x}\right) .
$$

The purpose of the present section is to prove the following proposition as a generalization of Proposition 3.4.

Proposition 4.1. If an infinitesimal conformal invariant variation of a compact orientable invariant submanifold of a Sasakian manifold is fiberpreserving, then it is isometric and hence f-preserving.

To prove this proposition we need following lemmas which will be proved in the same way as in the proofs of Lemmas 3.2 and 3.3.

LEMMA 4.2. In order for an infinitesimal conformal invariant variation of an invariant submanifold to be f-preserving, it is necessary and sufficient that the tensor field ' $T_{c b}$ defined by

$$
\begin{aligned}
{ }^{\prime} T_{c b}= & \nabla_{c} v_{b}-\left(\nabla_{e} v_{d}\right) f_{c}{ }^{e} f_{b}{ }^{d}-2 h_{c b}{ }^{x} v_{x}-f_{c} f_{b}\left(\nabla_{e} v_{d}\right) f^{e} f^{d} \\
& +f_{c}\left(f_{b}{ }^{e} v_{e}\right)-f_{b}\left(f_{c}{ }^{e} v_{e}\right)
\end{aligned}
$$

vanıshes identically.

LEMMA 4.3. For an infinttesimal conformal invariant variation of an $2 n$ variant submanifold we have

$$
\begin{gathered}
{ }^{\prime} T_{c b}+{ }^{\prime} T_{b c}=0 \\
{ }^{\prime} T_{c b}+{ }^{\prime} T_{e d} f_{c}{ }^{e} f_{b}{ }^{d}=\left(\mathcal{L} f_{c}\right) f_{b}-\left(\mathcal{L} f_{b}\right) f_{c}-2 \lambda f_{c} f_{b}
\end{gathered}
$$


and

$$
\begin{aligned}
{ }^{\prime} T^{c b \prime} T_{c b}= & 2^{\prime} T^{c b} \nabla_{c} v_{b}-2\left(\mathcal{L} f^{e}\right)\left(\mathcal{L} f_{e}\right) \\
& -4\left(\mathcal{L} f^{e}\right)\left(f_{e}{ }^{d} v_{d}\right)+2 \lambda f_{e}\left(\mathcal{L} f^{e}\right),
\end{aligned}
$$

where ${ }^{\prime} T^{c b}={ }^{\prime} T_{e d} g^{e c} g^{d b}$.

Proof of Proposition 4.1. Differentiating (4.1) covariantly, we have

$$
\nabla_{c} \nabla_{b} v_{a}+\nabla_{c} \nabla_{a} v_{b}=2 \nabla_{c}\left(h_{b a}{ }^{x} v_{x}+\lambda g_{b a}\right),
$$

from which, using the Ricci identity,

$$
\nabla_{c} \nabla_{b} v_{a}+\nabla_{a} \nabla_{c} v_{b}-K_{c a b d} v^{d}=2 \nabla_{c}\left(h_{b a}{ }^{x} v_{x}+\lambda g_{b a}\right),
$$

or, substituting (4.5),

$$
\nabla_{c} \nabla_{b} v_{a}-\nabla_{a} \nabla_{b} v_{c}-K_{c a b d} v^{d}=2 \nabla_{c}\left(h_{b a}{ }^{x} v_{x}+\lambda g_{b a}\right)-2 \nabla_{a}\left(h_{c b}{ }^{x} v_{x}+\lambda g_{c b}\right) .
$$

Taking the skew-symmetric part of this equation with respect to $a$ and $b$ and making use of the Ricci identity, we find

$$
\begin{aligned}
\nabla_{c} \nabla_{b} v_{a} & -\nabla_{c} \nabla_{a} v_{b}+K_{a b c d} v^{d}+K_{a c b d} v^{d}+K_{c b a d} v^{d} \\
& =-2 \nabla_{a}\left(h_{c b} x v_{x}+\lambda g_{c b}\right)+2 \nabla_{b}\left(h_{c a} v_{x}+\lambda g_{c a}\right),
\end{aligned}
$$

or, using (4.5) and the first Bianchi identity,

$$
\begin{aligned}
\nabla_{c} \nabla_{b} v_{a}-K_{b a c d} v^{d} & =\nabla_{c}\left(h_{b a}{ }^{x} v_{x}+\lambda g_{b a}\right)+\nabla_{b}\left(h_{c a}{ }^{x} v_{x}+\lambda g_{c a}\right) \\
& -\nabla_{a}\left(h_{c b}{ }^{x} v_{x}+\lambda g_{c b}\right)
\end{aligned}
$$

Transvecting this with $g^{c b}$ and using $h_{e}{ }^{e} x=0$, we find

$$
\nabla^{c} \nabla_{c} v_{a}+K_{a c} v^{c}-2 \nabla^{c}\left(h_{c a}{ }^{x} v_{x}\right)+(2 n-1) \nabla_{a} \lambda=0 .
$$

Now applying the operator $\nabla^{c}$ to (4.3) and using the Ricci identity, we can verify that

$$
\begin{aligned}
\nabla^{c \prime} T_{c b}= & \nabla^{c} \nabla_{c} v_{b}+\frac{1}{2} K_{c e d}{ }^{a} f^{c e} f_{b}^{d} v_{a}-2 \nabla^{c}\left(h_{c b}{ }^{x} v_{x}\right)-\left(\nabla^{e} \lambda\right) f_{e} f_{b}-v_{b} \\
& +(2 n+1)\left(f^{e} v_{e}\right) f_{b}+2 n\left(\nabla_{e} v_{d}\right) f^{e} f_{b}{ }^{d}
\end{aligned}
$$

because of (1.18), (1.20), (4.1) and $\left(\nabla_{e} v_{d}\right) f^{e} f^{d}=\lambda$.

Substituting (1.27) into this equation and taking account of (4.6), we obtain

$$
\nabla^{c}\left[{ }^{\prime} T_{c b}+2(n-1) \lambda g_{c b}+\lambda f_{c} f_{b}\right]=-2 n f_{b}^{c} \mathcal{L} f_{c} .
$$


Thus we have

$$
\begin{aligned}
\nabla^{c} & {\left[\left({ }^{\prime} T_{c b}+(2 n-1) \lambda g_{c b}+\lambda f_{c} f_{b}\right) v^{b}\right] } \\
& =-2 n\left(f_{b}^{c} v^{b}\right) \mathcal{L} f_{c}+\left[{ }^{\prime} T_{c b}+(2 n-1) \lambda g_{c b}+\lambda f_{c} f_{b}\right] \nabla^{c} v^{b},
\end{aligned}
$$

from which, substituting (4.4) and using (4.2) with $h_{e}{ }^{e}=0$,

$$
\begin{aligned}
\nabla^{c}[ & \left.\left({ }^{\prime} T_{c b}+(2 n-1) \lambda g_{c b}+\lambda f_{c} f_{b}\right) v^{b}\right] \\
= & \frac{1}{2}{ }^{\prime} T^{c b \prime} T_{c b}+4 n^{2} \lambda^{2}+\left(\mathcal{L} f^{e}\right)\left(\mathcal{L} f_{e}\right)+2(n+1)\left(\mathcal{L} f^{e}\right) f_{e}^{d} v_{d} \\
& -\lambda f_{e}\left(\mathcal{L} f^{e}\right) .
\end{aligned}
$$

The variation being conformal and fiber-preserving, from $\delta g_{c b}=2 \lambda g_{c b} \varepsilon$ and $g_{c b} f^{c} f^{b}=1$, we see that $\delta f^{a}=-\lambda f^{a} \varepsilon$, that is, $\mathcal{L} f^{a}=-\lambda f^{a}$ by (3.5). Thus the above equation becomes

$$
\nabla^{c}\left[\left({ }^{\prime} T_{c b}+(2 n-1) \lambda g_{c b}+\lambda f_{c} f_{b}\right) v^{b}\right]=\frac{1}{2}{ }^{\prime} T^{c b} T_{c b}+4 n^{2} \lambda^{2}
$$

Thus if the submanifold is compact and orientable, we have

$$
\int\left[{ }^{\prime} T^{c b^{\prime}} T_{c b}+8 n^{2} \lambda^{2}\right] d V=0
$$

from which $\lambda=0$, and consequently the variation is isometric and hence $f$-preserving.

\section{$\S 5$. An integral formula.}

We put

$$
S_{b}^{a}=\left(\nabla_{b} v^{e}\right) f_{e}^{a}-f_{b}^{e}\left(\nabla_{e} v^{a}\right)-2 h_{b}{ }^{e} v^{x} f_{e}^{a}+\left(f_{b} v^{a}-v_{b} f^{a}\right) .
$$

Then, as is shown in Lemma 3.1, an invariant variation of an invariant submanifold is $f$-preserving if and only if $S_{b}{ }^{a}=0$. We put $\left\|S_{c b}\right\|^{2}=S_{c b} S^{c b}$ where $S_{c b}=S_{c}{ }^{e} g_{e b}$ and $S^{c b}=g^{c e} S_{e}{ }^{b}$. Then, using (1.19) and (1.21), we obtain by a straightforward computation,

$$
\begin{aligned}
\left\|S_{c b}\right\|^{2}= & 2\left\|\nabla_{c} v_{b}\right\|^{2}-8\left(h_{e d}^{x} v_{x}\right) \nabla^{e} v^{d}+4\left\|h_{c b} v_{x}\right\|^{2} \\
& -\left\|\left(\nabla_{b} v_{e}\right) f^{e}\right\|^{2}-\left\|\left(\nabla_{e} v_{b}\right) f^{e}\right\|^{2}-2 f^{c b}\left(\nabla_{c} v^{e}\right)\left(\nabla_{b} v_{a}\right) f_{e}^{a} \\
& +2\left(f^{e} \nabla_{e} v^{b}\right) f_{b}^{a} v_{a}+2\left(f^{e} \nabla_{b} v_{e}\right) f_{a}^{b} v^{a}+2 v_{a} v^{a}-2\left(f_{a} v^{a}\right)^{2} .
\end{aligned}
$$


On the other hand, putting

$$
w^{b}=\left(\nabla^{b} v^{a}\right) v_{a}-f^{b a}\left(\nabla_{a} v_{c}\right) f_{e}^{c} v^{e}+\left(f_{a}^{b} v^{a}\right)\left(f^{e} v_{e}\right),
$$

we have

$$
\begin{aligned}
\nabla_{b} w^{b}= & \left(\nabla^{b} \nabla_{b} v_{a}\right) v^{a}+\left\|\nabla_{b} v_{a}\right\|^{2}-f^{c b}\left(\nabla_{c} \nabla_{b} v_{a}\right) v^{e} f_{e}{ }^{a}-f^{c b}\left(\nabla_{c} v^{e}\right)\left(\nabla_{b} v_{a}\right) f_{e}^{a} \\
& +2 n\left(f^{e} \nabla_{e} v_{d}\right) f_{c}{ }^{a} v^{c}+2 f^{e}\left(\nabla_{d} v_{e}\right) f_{c}{ }^{d} v^{c}+(2 n+1)\left(f_{e} v^{e}\right)^{2}-\left(v_{e} v^{e}\right),
\end{aligned}
$$

from which, using (1.27) and the Ricci identity,

$$
\begin{aligned}
\nabla_{b} w^{b}= & v^{a}\left(\nabla^{b} \nabla_{b} v_{a}+K_{b a} v^{b}\right)+\left\|\nabla_{b} v_{a}\right\|^{2}-f^{c b}\left(\nabla_{c} v^{e}\right)\left(\nabla_{b} v_{a}\right) f_{e}^{a} \\
& +2 n\left(f^{e} \nabla_{e} v_{d}\right) f_{c}{ }^{d} v^{c}+2 f^{e}\left(\nabla_{d} v_{e}\right) f_{c}{ }^{d} v^{c}-2 n\left(v_{e} v^{e}\right)+2 n\left(f_{e} v^{e}\right)^{2} .
\end{aligned}
$$

Comparing (5.1) with (5.2), we have

$$
\begin{aligned}
\left\|S_{c b}\right\|^{2}= & 2 \nabla^{b} w_{b}-2 v^{a}\left(\nabla^{b} \nabla_{b} v_{a}+K_{b a} v^{b}\right)-8\left(h_{b a}{ }^{x} v_{x}\right) \nabla^{b} v^{a}+4\left\|h_{c b}{ }^{x} v_{x}\right\|^{2} \\
& +(4 n+2)\left\{v_{e} v^{e}-\left(f_{e} v^{e}\right)^{2}+\left(f^{e} \nabla_{e} v^{d}\right) f_{d}{ }^{c} v_{c}\right\}-2 f^{e}\left(\nabla_{d} v_{e}\right) f_{c}{ }^{d} v^{c} \\
& -\left\|\left(\nabla_{b} v_{e}\right) f^{e}\right\|^{2}-\left\|\left(\nabla_{e} v_{b}\right) f^{e}\right\|^{2},
\end{aligned}
$$

or equivalently

$$
\begin{aligned}
\left\|S_{c b}\right\|^{2}= & 2 \nabla^{b}\left(w_{b}-2 h_{b a}{ }^{x} v_{x} v^{a}\right)-2 v^{a}\left[\nabla^{b} \nabla_{b} v_{a}+K_{b a} v^{b}-2 \nabla^{b}\left(h_{b a} v_{x}\right)\right] \\
& -2\left(h^{c b}{ }_{y} v^{y}\right)\left(\nabla_{c} v_{b}+\nabla_{b} v_{c}-2 h_{c b}{ }^{x} v_{x}\right) \\
& -\left\|\mathcal{L} f_{a}\right\|^{2}-\left\|\mathcal{L} f^{a}\right\|^{2}-4(n+1)\left(\mathcal{L} f^{e}\right) f_{e}{ }^{d} v_{d} .
\end{aligned}
$$

Therefore, assuming that the submanifold is compact orientable, we apply Green's theorem and obtain

$$
\begin{aligned}
& \int\left[\frac{1}{2}\left\|S_{c b}\right\|^{2}+v^{a}\left\{\nabla^{b} \nabla_{b} v_{a}+K_{b a} v^{b}-2 \nabla^{b}\left(h_{b a}{ }^{x} v_{x}\right)\right\}\right. \\
& \quad+\left(h_{y}^{c b} v^{y}\right)\left(\nabla_{c} v_{b}+\nabla_{b} v_{c}-2 h_{c b}{ }^{x} v_{x}\right) \\
& \left.\quad+\frac{1}{2}\left(\left\|\mathcal{L} f_{a}\right\|^{2}+\left\|\mathcal{L} f^{a}\right\|^{2}\right)+2(n+1)\left(\mathcal{L} f^{e}\right) f_{e}^{d} v_{d}\right] d V=0 .
\end{aligned}
$$

From (2.12) and (2.13) we see that the infinitesimal variation of $d V$ is given by

$$
\delta d V=\left(\nabla_{a} v^{a}-h_{a}{ }^{a}{ }_{x} v^{x}\right) d V \varepsilon .
$$


On the other hand it is known [2] that the integral formula

$$
\int\left[v^{a}\left(\nabla^{b} \nabla_{b} v_{a}+K_{b a} v^{b}\right)+\frac{1}{2}\left\|\nabla_{b} v_{a}+\nabla_{a} v_{b}\right\|^{2}-\left(\nabla_{e} v^{e}\right)^{2}\right] d V=0
$$

is valid for any vector field $v^{a}$ in a compact orientable Riemannian manifold. From this we have

$$
\begin{aligned}
& \int\left[v^{a}\left\{\nabla^{b} \nabla_{b} v_{a}+K_{b a} v^{b}-2 \nabla^{b}\left(h_{b a}{ }^{x} v_{x}\right)+\nabla_{a}\left(h_{e}^{e} x v^{x}\right)\right\}\right. \\
& \quad+\frac{1}{2}\left\|\nabla_{b} v_{a}+\nabla_{a} v_{b}-2 h_{b a}{ }^{x} v_{x}\right\|^{2}-\left(\nabla_{e} v^{e}-h_{e}^{e}{ }_{x} v^{x}\right)\left(\nabla_{b} v^{b}\right) \\
& \left.\quad+\left(h_{y}^{c b} v^{y}\right)\left(\nabla_{c} v_{b}+\nabla_{b} v_{c}-2 h_{c b}{ }^{x} v_{x}\right)\right] d V=0 .
\end{aligned}
$$

Comparing (5.3) with (5.5) and taking account of $h_{e}{ }^{e} x=0$, we have

$$
\begin{aligned}
& \int\left[\left\|S_{c b}\right\|^{2}-\left\|\nabla_{c} v_{b}+\nabla_{b} v_{c}-2 h_{c b}{ }^{x} v_{x}\right\|^{2}+2\left(\nabla_{e} v^{e}\right)^{2}\right. \\
& \left.\quad+\left\|\mathcal{L} f_{a}\right\|^{2}+\left\|\mathcal{L} f^{a}\right\|^{2}+4(n+1)\left(\mathcal{L} f^{e}\right) f_{e}^{d} v_{d}\right] d V=0,
\end{aligned}
$$

or using $S_{e}^{e}=g^{e d} S_{e d}=0$,

$$
\begin{gathered}
\int\left[\left\|\nabla_{c} v_{b}+\nabla_{b} v_{c}-2 h_{c b}{ }^{x} v_{x}\right\|^{2}-\left\|S_{c b}+\sqrt{2 /(2 n+1)}\left(\nabla_{e} v^{e}\right) g_{c b}\right\|^{2}\right. \\
\left.+\left\|\mathcal{L} f_{a}\right\|^{2}+\left\|\mathcal{L} f^{a}\right\|^{2}+4(n+1)\left(\mathcal{L} f^{e}\right) f_{e}{ }^{d} v_{d}\right] d V=0 .
\end{gathered}
$$

Now, when we have $\delta f^{a}=\left(\mathcal{L} f^{a}\right) \varepsilon=0$, we say that the variation is structly fiber-preserving. From (5.6) we have

PROPOSITION 5.1. In order for an infinitesimal strictly fiber-preserving invariant variation of a compact orientable invariant submanifold of a Sasakian manifold to be isometric, it is necessary and sufficient that the variation is volume-preserving and f-preserving.

Now if an infinitesimal variation is affine we have from (2.19) $\nabla_{c}\left(\nabla_{e} v^{e}\right)=0$, since $h_{e}{ }^{e}=0$. Thus $\nabla_{e} v^{e}=$ const. and consequently if the submanifold is compact we have $\nabla_{e} v^{e}=0$. Thus from Proposition 5.1, we have

Proposition 5.2. A strictly fiber-preserving invariant variation of a compact orientable submanifold of a Sasakıan manifold is isometric if and only if the variation is affine and f-preserving. 
Remark. From (5.6) we see that if a fiber-preserving variation of a submanifold is conformal, then $\lambda=0$ and $S_{c b}=0$. This gives another proof of Proposition 4. 1 .

\section{BIBLIOGRAPHY}

[1] S. TAchibana, On the isometric deformation vector of complex hypersurface, to appear.

[2] K. YANO, Differential geometry on complex and almost complex spaces, Pergamon Press, (1965).

[3] K. YANO, Infinitesimal variations of submanifolds, Kōdai Math. J., 1 (1978), $30-44$.

[4] K. Yano and S. TACHibana, Sur les déformations cumplexes isométriques des hypersurfaces complexes, C. R. Acad. Sci. Paris, 282 (1976), 1003-1005.

[5] K. YANO, U-HANG KI and M. OKUMURA, Infinitesimal variations of invariant submanifolds of a Kaehlerıan manifold, Kōdai Math. J., 1 (1978), 89-100.

TOKyo Institute of Technology

AND

KYUNGPOOK UNIVERSITY 